\title{
Depoimento
}

\section{Visar ao sucesso dos alunos: a ortopedagogia e a alfabetização}

\section{To aim students' success: orthopedagogie and literacy Viser la réussite des élèves: l'orthopédagogie et l'alphabétisation}

Stéphanie COTE

\section{RESUMO}

Este depoimento fala da minha experiência profissional no campo da Ortopedagogia. A parceria entre ortopedagogos, alfabetizadores, demais profissionais da escola e da família é fundamental para o sucesso escolar dos alunos.

Palavras-chave: ortopedagogia, alfabetização, sucesso escolar.

\section{ABSTRACT}

This report speaks of my professional experience in orthopedagogie's field. The partnership between orthopedagogie's professionals, literacy teachers, students' families is the base for students's scess at school.

Index terms: orthopedagogie, literacy, school success.

\section{RESUME}

Ce témoignage parle de mon expérience professionnelle dans le champ de l'orthopédagogie. Le partenariat entre les orthopédagogues, les alphabétisateurs, les familles est fondamental au succès scolaire des élèves.

Mots clés: orthopédagogie, alphabétisation, succès scolaire. 
Tout d'abord, lorsque je travaille avec les jeunes de mon école, je le fais toujours en partenariat avec les enseignants (es). Ce que je veux dire c'est que je discute souvent avec eux pour savoir où ils sont rendus avec leurs élèves, ce qu'ils font présentement en classe, etc. Mon objectif lors de ces discussions est de travailler dans le même sens qu'eux, en collaboration avec eux. Nous ne devons pas se le cacher, notre but est le même pour tous: «Viser la réussite des élèves ».

Je travaille en petits groupes allant de 5 à 7 élèves afin de revoir des notions non acquises et leur permettre d'accéder à des connaissances plus avancées. Malheureusement, les enseignants ne peuvent pas toujours revenir sur une même notion si cette dernière n'est pas maîtrisée pour 5 ou 6 élèves dans la classe. Dans ce sens, les services en orthopédagogie sont complémentaires à tout ce que font les enseignants dans leur classe. Lorsque j'arrive dans une étude de cas, j'appuie souvent les dires de l'enseignant, j'apporte ma touche personnelle en nommant des choses acquises ou non par l'élève et mes évaluations réalisées avec lui donnent souvent des pistes d'intervention efficaces et en lien avec ses problématiques. D'ailleurs, dernièrement, j'ai eu une tâche assez difficile à régler. En fait, la psychologue de l'école a fait une évaluation détaillée d'un élève. Ce dernier a coté avec un déficit de l'attention, il a une dysphasie sévère et madame recommandait de le placer dans le fond de la classe afin qu'il cesse de déranger les autres... $\mathrm{OH}$ ! Je n'étais vraiment pas contente, j'ai dû lire le dossier de l'élève en profondeur et me « lever » pour lui afin de défendre ses droits. Ce n'est pas de sa faute s'il a des difficultés, qu'il n'est pas médicamenté et qu'il gère mal ses émotions... Il a un déficit de l'attention, ce qui veut dire que ses fonctions exécutives ne fonctionnent pas comme les autres... Je crois que c'est mon mandat de défendre cet enfant et de me battre afin d'expliquer l'ampleur du problème à l'équipe-école et par le fait même, dire tout haut ce dont il a besoin... Les élèves qui ont des difficultés 
ou des troubles spécifiques ne peuvent malheureusement pas toujours suivre correctement en classe. Ils ont souvent besoin de visuel, d'enseignement individualisé (1-1) et de choses concrètes pour mieux comprendre. Ainsi, s'il n'y avait pas d'enseignant en orthopédagogie dans les écoles, ces élèves ne pourraient pas avoir un tel suivi, un aussi beau service pour les aider. Nous sommes complémentaires aux enseignants du régulier parce que nous avons une formation spécialisée pour les troubles d'apprentissage, de la lecture et de l'écriture.

Je pense que pour me positionner en tant que coordonnateur et leader, il est important que je dise comment je vois le rôle de l'enseignant en orthopédagogie comme leader de l'éducation. Tout d'abord, je dois l'admettre, je ne trouve pas cela toujours facile de jouer ce rôle de leader dans une école. Quand tu arrives dans une nouvelle école et que tu viens de terminer l'université, c'est encore plus ardu. « La petite jeune qui sort de l'école ! » Il faut savoir mettre nos gants blancs et ne pas arriver en voulant tout changer d'un seul coup ! Nous devons d'abord et avant tout connaître les enseignants et leur manière de fonctionner. Mon âge et mon expérience nuisent parfois dans l'exercice de mes fonctions. Dans ce domaine, il ne faut pas être trop timide et savoir proposer quelques pistes d'intervention intéressantes. La rigidité, la fermeture et la défensive des enseignants sont parfois des embûches qui nous empêchent d'exercer notre leadership adéquatement. Comme un enseignant m'a déjà dit, le « timing » est un aidant incroyable. Quand quelque chose arrive et que tout s'organise adéquatement, c'est tout à fait extraordinaire. Pour exercer un bon leadership dans une école, il est essentiel d'avoir des alliés et encore mieux, la direction comme allié principal. De plus, il faut savoir se respecter, rester soi-même et se faire confiance. Lorsque toutes ses aptitudes sont présentes, nous pouvons aller loin, proposer de bonnes activités et devenir un leader à la hauteur de la situation. 
Un jour je me suis justement questionnée sur l'importance d'un orthopédagogue dans une école. Les fameux spécialistes des difficultés d'apprentissage... Sans nous, les élèves seraient parfois laissés à euxmêmes, feraient des activités bien différentes de celles des autres, ne seraient pas autant « intégrés », leur style d'apprentissage respectif ne serait pas pris en compte et je pense qu'il y aurait encore plus de décrochage scolaire. Les élèves seraient démunis et les enseignants, débordés. Je crois que nous avons beaucoup à apporter aux enseignants et ces derniers ont beaucoup à nous apporter également. Comme nous sommes les spécialistes des difficultés et de l'intégration, nous devons être en mesure de répondre aux questions des enseignants, de justifier nos actions et de promouvoir l'intégration des élèves ayant diverses difficultés. Dans ce sens, les attitudes des enseignants à notre égard nous aident positivement ou négativement à développer notre rôle de leadership. Quand les enseignants nous font confiance et que nous nous sentons appréciés, c'est deux fois plus facile de faire notre place, d'exposer nos idées et d'exercer notre rôle à pleine capacité !

À mon avis, la première grande responsabilité de l'enseignant en orthopédagogie est de faire ou encore de recevoir des références d'élèves pour ensuite ouvrir des dossiers au besoin et faire les évaluations adaptées aux besoins de chaque élève. Comme Paulo Freire (1987) le disait si bien, chaque être humain est un être différent, un sujet doué de conscience critique.

Voici maintenant ma description personnelle des responsabilités impliquées pour chacune des tâches du modèle 30-30-30.

Interventions directes :

Lors des interventions directes, l'enseignant soutien (orthopédagogue) doit jouer différents rôles de spécialistes. Il doit, entre autres, être celui des difficultés d'apprentissage, de l'évaluation et des acquis des élèves. De plus, nous devons être doués dans l'utilisation de 
diverses stratégies en mathématique, en français et nous nous devons de faire la prévention dans les classes. Dans ce sens, les responsabilités sont de:

Recevoir les jeunes, de manière individuelle ou en sous-groupe, dans un local.

- $\quad$ Faire l'évaluation complète, à l'aide de tests diagnostiques appropriés, en lecture en mathématique et en écriture afin de découvrir les acquis et les difficultés que vivent les élèves.

- $\quad$ Aider l'enseignant dans sa classe, et par le fait même, faire de la prévention et détecter les difficultés d'apprentissage chez certains.

- Animer des activités dans les classes en collaboration avec le titulaire.

- Revoir les notions qui ont été vues en classe et qui ne sont pas acquises par les élèves.

- $\quad$ Doter les élèves de stratégies pouvant les aider à résoudre des problèmes en mathématique et/ou en français (possibilités de faire des transferts).

Dans différentes écoles, l'enseignant soutien peut être appelé à intervenir directement auprès des élèves ayant des troubles de comportement.

Interventions indirectes et collaboration avec les enseignants:

Dans ce type d'intervention, l'enseignant soutien doit être un conseiller expert, un soutien à l'enseignant et un spécialiste de l'adaptation. Dans cette optique, les responsabilités que nous avons sont de:

- Préparer du matériel et/ou offrir du support à l'enseignant afin de les aider à faire de la différenciation pédagogique et à avoir plus de facilité à intégrer certains élèves (ex. TED).

Conseiller et outiller les enseignants de manière ponctuelle.

spécialisées.

Discuter avec les éducatrices

- Soutenir les enseignants lorsqu'ils ont des problèmes avec des élèves ayant des troubles d'apprentissage et/ou de comportement (dans certains cas).

- Observer les élèves dans leur classe afin de noter des faits importants.

Équipe-école, Équipe-cycle, Équipe-parents : 
Pour ce dernier 30\%, l'enseignant soutien doit être omniprésent dans divers comités et être un spécialiste des informations par rapport aux difficultés d'apprentissage. Dans ce sens, il doit :

- Assister à des plans d'intervention et/ou des études de cas.

- Discuter et regarder les dossiers des élèves avec la psychologue et/ou le psychoéducateur, la direction, l'équipe-école...

- $\quad$ Faire des activités / cycle avec tous les enseignants et les élèves (projet éducatif).

- $\quad \hat{t r e}$ présent, lors des rencontres des parents, afin de recevoir ceux qui désirent avoir des informations supplémentaires par rapport aux services offerts.

Voici donc un bref portrait de mes croyances et de ma conception actuelle de ce qu'est un enseignant orthopédagogue. Je crois qu'en exposant ma manière d'être et de penser en tant qu'orthopédagogue dans une école primaire, il est maintenant facile de voir pourquoi mon rôle est un « service complémentaire essentiel à la réussite » des élèves.

Jean Biarnès (1999) disait que la première mission de l'orthopédagogue est d'instruire, de former et d'accompagner la dimension créatif des éléves..." Je crois qu'il est très important de travailler avec le jeune, de lui montrer de nouvelles manières de faire les choses mais non de le faire à sa place. À mon avis, une bonne façon d'aller toucher les enfants c'est d'utiliser le concret, de leur faire vivre des émotions et leur faire créer des images dans leur tête...

\title{
Réferénces bibliographiques
}

\author{
BIARNÈS, Jean (1999). Universalité, diversité, sujet dans l'espace \\ pédagogique. Paris: L'Harmattan.
}

FREIRE, Paulo (1987). Pedagogia do oprimido. Rio de Janeiro: Paz e Terra. 


\section{Autora:}

Stéphanie Côté

Étudiante en D.E.S.S. en Orthopédagogieau à l'Université du Québec à Chicoutimi (UQAC).

Contato: - stéphabie.cote@uqac.ca

\section{Como citar este depoimento:}

\section{Como citar este artigo:}

COTE, Stéphanie. Visar ao sucesso dos alunos: a ortopedagogia e a alfabetização. Revista ACOALFAplp: Acolhendo a Alfabetização nos Países de Língua portuguesa, São Paulo, ano 5, n. 9, 2010/ 2011. Disponível em: <http://www.acoalfaplp.net>. Publicado em: setembro de 2010 - março de 2011.

Recebido em abril de 2009./ Aprovado em junho de 2009.

Veja aqui algumas imagens da cidade do Quebec. 